\title{
Basic Principles in Carrying Out a Pilot Therapeutic Trial in Leprosy
}

\author{
W. H. JOPLING, F.R.C.P.E., M.R.C.P., D.T.M.\&H. \\ Jordan Hospital, Redhill, Surrey, and The Hospital for Tropical Diseases, London N.W. I.
}

I A TRIAL OF A NEW ANTI-LEPROSY DRUG

\section{The Patients}

Patients for the trial should be suffering from lepromatous leprosy as this is the type of leprosy which responds most poorly and most predictably to standard treatment, lesions are plentiful, and acid-fast bacilli are present in large numbers. Borderline (dimorphous, intermediate or bipolar) cases are less suitable as their progress on standard treatment is more variable and less predictable, and the numbers of bacilli in skin lesions are less plentiful and consistent. Tuberculoid cases are unsuitable as the diagnosis is not always easy to establish, and the fact that bacilli are absent from skin lesions makes progress difficult to assess.

Patients should not have had previous treatment. This does not apply to those who were treated several years previously and have relapsed after stopping treatment, but to those who are already under treatment at the time of commencing the trial.

Finally, the trial should be carried out on in-patients, thus making it easier to supervise treatment, to make regular assessments of progress, and to discover any complications. One cannot be sure that out-patients are taking tablets as directed or that they will report at regular intervals for examination.

\section{Preliminary Tests}

A careful record must be made of the appearance and distribution of skin lesions, the presence of thickened peripheral nerves, and the extent of any sensory impairment. Visual acuity in each eye should be recorded; a patient who does not understand letters of the alphabet can be shown drawings of well known animals or household objects, and testing charts of this type can be made if they cannot be obtained. The eyes should be examined with a torch and corneal loupe for the presence of superficial punctate keratitis, and this is important as corneal changes are slow to clear on standard sulphone therapy and may even become worse.

General medical examination should include a record of weight and height, and the following tests are obligatory: Blood count (haemoglobin, red blood cells, white cells and differential), examination of the blood for malarial or other parasites, urine and stool examination, and chest X-ray. Any intercurrent disease should be treated before the patient enters the trial.

Eight skin smears are made in order to estimate the Bacterial Index and to note the morphology of the bacilli. Smears are taken from the two ear lobes and from six activelooking skin lesions on various parts of the skin; these six sites are carefully marked on a chart so that future smears will be made from the same lesions. Nasal smears are not recommended. An increasing proportion of fragmented and granular bacilli during the course of treatment is a valuable index of improvement even if the total quantity of acid-fast material in smears remains unchanged. There are several systems in use for recording the Bacterial Index, and the one used at the Jordan Hospital is that described by Ridley (1958) :

$6+$ more than rooo bacilli in an average oilimmersion field.

$5+$ roo- Iooo bacilli in an average oilimmersion field.

$4+$ Io- I oo bacilli in an average oil-immersion field.

3 t- I-Io bacilli in an average oil-immersion field.

$2+$ I-Io bacilli, on average, in ro fields.

I + I-Io bacilli, on average, in roo fields.

For recording the morphology of bacilli in smears we use the Granularity Index designed by Ridley ( 1964$)$. 
A skin biopsy is carried out to establish the type of leprosy and to assess the heaviness of the infection (see Ridley's papers, mentioned above, for a description of the Biopsy Index). It is desirable to choose a raised, plaque-like lesion for biopsy, and, if possible, one that is large enough to enable further biopsies to be taken from other parts of it during the course of the trial. In the unusual event of there being no lesion large enough for this, a careful note should be made of the position of at least six skin lesions of similar size and consistency so that they can be removed during the course of the trial. My method is to biopsy two different lesions in order to reduce the chances of error, and we take the mean of the two Biopsy Indices. I use a skin biopsy punch with a circular cutting edge $5 \mathrm{~mm}$. in diameter, and this is driven down to the subcutaneous tissue with a rotatory boring action after I - $2 \mathrm{ml}$. of I \% lignocaine hydrochloride mixed with hyalase (hyaluronidase) has been injected into the centre of the portion of skin to be removed. Where facilities for histological work do not exist, arrangements can be made to send tissue by Air to a centre where sections can be cut and examined, e.g. to Dr D. S. Ridley's laboratory at the Hospital for Tropical Diseases in London, or to Dr R. G. Cochrane's Leprosy Research Unit at $57 \mathrm{a}$ Wimpole Street, London, W.I. The tissue can be sent in a suitable fixative.

The lepromin test is not essential if the trial is being confined to lepromatous cases as the reaction will remain negative throughout.

Photographs should be taken to show the extent and distribution of the lesions, and should include a front view of the face and two side views to include the ears.

\section{Details of the Trial}

As soon as it has been decided to carry out a trial, all lepromatous cases admitted to hospital after that date are included and each patient is given an ordinary hospital number and also a special number for the trial ('T.I.', 'T.2.', 'T.3.', etc.). All those with odd numbers in the ' $\mathrm{T}$ ' series are given sulphone alone, and those with even numbers receive sulphone plus the trial drug, thus avoiding bias in the selection of cases. As both groups of patients receive a proven anti-leprosy drug no ethical problem is raised. All patients should receive the same sulphone on a predetermined dosage scheme, and parenteral sulphone is preferable to oral. The number of patients in the trial will depend on the number available, and, although larger numbers are desirable, as few as io would give useful information about a new drug. The co-operation of an independent assessor (who would not know which patients were receiving the trial drug) would be an advantage.

Observations on both groups should include: (a) Daily Observations: Morning and evening recordings of body temperature.

(b) Weekly Observations: Clinical: Notes should be made on the condition of the patients, with special reference to the appearance of skin lesions, their colour on inspection and their consistency on palpation. Peripheral nerves should be palpated for any change in thickness or any tenderness. Weight and visual acuity should be recorded. Pathological: Haemoglobin and total white cell count. Urine examination for protein and sugar.

(c) Monthly Observations: A record should be made of any change in keratitis (if present), and skin sensation is tested for any increase or decrease in anaesthesia.

(d) Three-monthly Observations: Photographs are taken, and these should be comparable with the original ones as regards body sites, lighting, distance, and in all other respects. Eight smears are made from the sites previously used.

(e) Six-monthly Observations: Two skin biopsies are carried out.

\section{Problems arising during the course of the Trial}

Treatment need not be interrupted for an intercurrent infection, but a more difficult problem is posed by the development of a lepra reaction (E.N.L. or Type II reaction - Jopling, I959). The ideal solution is to continue antileprosy treatment and to use all available means, including steroids where necessary, to control the reaction. Waters (1963) in his trial of macrocyclon adopted this policy with success. A lepromatous exacerbation (Type I reaction Jopling, I 959) does not call for any reduction in dosage of anti-leprosy drug or for any special treatment. If the trial drug produces a toxic effect such as anaemia, granulopenia, or proteinuria, the saf est course of action would be to abandon the trial. In such an event a note should be published without delay in a Leprosy Journal drawing attention to the drug's toxicity. 
2 A TRIAL OF A DRUG TO GONTROL LEPRA REACTION

In order to avoid bias in the selection of cases and to supply a control series for comparison with the trial drug, the first patient to undergo a lepra reaction is numbered 'T.I', the second 'T.2.', and so on. Those with odd numbers receive a placebo and those with even numbers receive the anti-inflammatory drug. As there may be objections to giving an inert drug to patients suffering from reaction, the best plan is to give two aspirin tablets thrice daily to those in the control series, and the effect of the trial drug can be compared with that of aspirin. A rule must be made from the very beginning of the trial on the question of whether to continue or stop the anti-leprosy drug when reactions occur, and the safest plan to adopt is to stop it as soon as the trial drug (or the aspirin, as the case may be) is commenced.

\section{REFERENCES}

JOPLing, W. H. (I959). Correspondence - Reactional Leprosy. Leprosy Rev. 3o, I 94-I 96.

RIDLEY, D. S. (I958). Therapeutic trials in leprosy using serial biopsies. Leprosy Rev., 29, 45-52.

RIDLEy, D. S. (1964). Leprosy in Theory and Practice, pp. 620622, ed Cochrane, R. G. and Davey, T. F., Bristol: John Wright \& Sons Ltd.

WATERs, M. F. R. ( I 963). Chemotherapeutic trials in leprosy. Leprosy Rev., 34, I 73-I $9^{2}$. 